\title{
Estrés y actitudes resilientes en el personal de la Policía Nacional del Perú frente a la pandemia causada por el covid-19
}

Stress and resilient attitudes in the personnel of the National Police of Peru in the face of the pandemic caused by covid-19 Recepción del artículo: 19-02-2021 ｜ Aceptación del artículo: 28-06-2021

Román Jesús, Marquina-Lujan Universidad San Ignacio de Loyola (Perú)

rmarquina@usil.edu.pe

https://orcid.org/0000-0002-5542-7234

Alexander Masías Benavides Román Universidad César Vallejo (Perú)

benavidesro22@ucvvirtual.edu.pe https://orcid.org/0000-0003-0398-938X
Para referenciar este artículo:

Marquina-Lujan, R. J. y Benavides-Román, A. M. (2021). Estrés y actitudes resilientes en el personal de la Policía Nacional del Perú frente a la pandemia causada por el covid19. Revista ConCiencia EPG, 6(2), 132-142. https://doi.org/10.32654/CONCIENCIAEP

\section{Resumen}

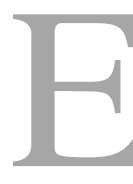

l objetivo del presente estudio busca establecer la relación que existe entre la capacidad resiliente $y$ el estrés que presentan los efectivos policiales que se desempeñan en la ciudad de Lima en tiempos de COVID-19. Para el presente estudio se utilizó la escala de resiliencia de 14 ítems (RS-14) la cual presenta una confiabilidad mediante alfa de Cronbach de .787 y para medir el estrés se elaboró un cuestionario en relación a la sintomatología que desarrolla una persona estresada en su dimensión física, psicología y conductual, encontrando una fiabilidad de .912 La muestra estuvo conformada por 408 efectivos policiales en actividad, de los cuales son 219 varones y 189 mujeres. Los resultados nos indican que existe relación entre la actitud resiliente y el estrés en los agentes policiales de Lima Metropolitana frente a la pandemia causada por la presencia del COVID-19. Se concluye que la capacidad resiliente ubicada en un nivel medio permite adaptarse a las exigencias del contexto y tener un desempeño adecuado, a pesar de los niveles alto de estrés encontrados.

Palabras Clave: Resiliencia, estrés, covid-19, Policía Nacional del Perú. 
Abstract

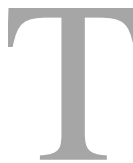

he purpose of the current research is to establish the relation between resilience capacity and the stress that police force officers who serve in Lima feel during the COVID-19 pandemic. For this study a resilience scale of 14 items (RS-14) was utilized. This scale shows an alpha Cronbach of .787, and to measure stress, a questionnaire about symptoms that a person under stress feels, and the physical, psychological, and behavioral dimensions that accompany that stress, was elaborated; with an alpha Cronbach of .912. The sample included 219 men and 189 women. The results show that there is a relation between resilience attitude and stress in police officers who face the COVID-19 pandemic. It is concluded that the resilient capacity located at a medium level, allows adapting to the demands of the context and having an adequate performance, despite the high levels of stress encountered.

Key Words: Resilience, stress, covid-19, Peruvian Police Force.

\section{Introducción}

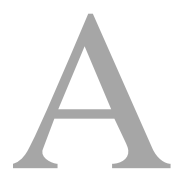

ctualmente, el mundo entero vive las consecuencias más letales que una enfermedad ha podido causar desde el año 1918 con la pandemia de influenza H1N1 que cobro la vida de más de 50 millones de personas en el mundo. Nos referimos al COVID-19 descubierta el mes de diciembre del 2019 en la provincia de Wuhan (China), enfermedad contagiosa causada por el coronavirus y que afecta a miles de seres humanos en todo el orbe, esta pandemia, declarada así por distintos organismos internacionales, viene afectando, además, la economía, los servicios públicos y privados, la estabilidad laboral y la salud mental de las personas, entre otros (OMS, 2021). En el Perú, a través del Decreto Supremo N0442020-PCM del 15 de marzo del 2020, se declaró el Estado de Emergencia Nacional, estableciéndose el aislamiento social obligatorio (cuarentena) y la suspensión del ejercicio de los derechos constitucionales (El Peruano, 2020), evidenciándose con dichas medidas que el virus era letal para la salud y se tenía que evitar su propagación.

Las muertes y el número de contagios, han ido en aumento desde que se presentó el COVID-19 y sus variantes; y son los profesionales de la salud, las Fuerzas Armadas y los miembros de la Policía Nacional del Perú, quienes cumplen un rol preponderante al ponerse al frente y en primera línea; no solo en la atención y tratamiento de las personas que se contagian; sino, en la prevención de la propagación del mismo en la contención de ciudadanos que incumplen con las medidas adoptadas por el gobierno.

El Banco Mundial (2020) señala en uno de sus informes que la crisis vivida por la presencia del COVID-19 podría ser comparada con la crisis financiera mundial de los años 2007 y 2008 y con las consecuencias que trajo consigo la segunda 
guerra mundial, cuyas secuelas se conocerán más adelante. En este contexto el Perú ha sido una de los países que mayor impacto ha causado esta enfermedad.

Una de las instituciones más afectadas por el COVID-19 fue la Policía Nacional del Perú (PNP), ente tuitivo del Estado, que tiene como finalidad fundamental el de prestar ayuda $y$ protección a los ciudadanos, a la comunidad y garantizar, entre otras funciones, el orden interno y el cumplimiento de las leyes (Constitución, 1993), por lo que la labor que cumple la PNP es indispensable para que los ciudadanos y la sociedad en general acaten las disposiciones del toque de queda, aislamiento social y otros decretadas por el supremo gobierno; implicando que los efectivos policiales en el desarrollo de sus actividades y en el marco de la pandemia estén en contacto directo con personas que no cumplen la ley, presentando un mayor riesgo de contagio en sus intervenciones y más aún cuando se producen detenciones de personas que se rehúsan o se oponen a ella, generando el uso legal de la fuerza (contacto físico) en su traslado a la dependencia policial.

La PNP ha sido la institución más expuesta en la ciudad para contagiarse del coronavirus, sus principales funciones de hacer cumplir la ley y de garantizar y mantener el orden público ha implicado una mayor interacción con la población (IDL, 2020). El Ministerio del Interior, a través del equipo de trabajo de la red de seguridad del personal PNP. ETRSP PNP COVID-19 en su Boletín Diario (2021) informó que, al ocho de febrero del 2021, ya eran 573 agentes policiales los fallecidos por el virus y 42, 669 casos habían sido reportados como positivos; es decir, un poco más del $30 \%$ de la población policial a nivel nacional; situación que de alguna manera merma la moral y capacidad operativa de los miembros policiales.

En definitiva, se puede fácilmente pensar que la labor de un policía, demanda el desarrollo de muchas habilidades personales, como la responsabilidad social, el ser asertivo, la empatía, el liderazgo, el lidiar en su lucha incesante contra la delincuencia, entre otros; pero, consideramos que en el contexto que vivimos actualmente, debido a la vulnerabilidad que acarrea la presencia del COVID-19, demanda mucho, la actitud resiliente y el manejo del estrés, de parte de este personal, que labora en primera línea.

Es indudable que la pandemia ha generado una transformación en los seres humanos reflejado en el cambio de actitudes, hábitos, costumbres y de interacción, sometiéndose a reacciones para posibilitar la preservación de su estado de salud ante la amenaza real existente (Tirino-Quijije \& Giniebra-Urra, 2020). En este sentido los efectivos de la PNP no son ajenos a esta situación a pesar de su formación semi castrense.

Lo que se debe resaltar, frente a toda la crisis o situación problema que se presenta en algún momento de nuestras vidas, es que el ser humano tiene la capacidad de poder enfrentar las diversas crisis que se le puedan presentar y salir airosos de ella, basado en la autoconfianza y su autonomía (Marquina-Luján, 2017). Esta capacidad es conocida como 
resiliencia, la cual es relevante en el estudio del comportamiento humano, caracterizado por la constancia, que tiene la persona para enfrentar situaciones adversas. Becoña (2006) refirió que muchos autores han encontrado que esta desarrolla una habilidad exitosa para afrontar al estrés.

De acuerdo a Rutter (2007) la resiliencia importa la disminución de los riesgos frente a las situaciones ambientales que se presentan $y$ que ante dicha vulnerabilidad se sobreponga al estrés o adversidad para obtener resultados positivos. Las personas resilientes siempre buscan adaptarse a nuevas situaciones, que se van desarrollando con cambios diversos, buscando la moderación entre el organismo y el espacio donde interactúa, causando distintitos niveles de estrés en ellos (Águila, Calcines, Monteagudo y Nieves (2015). En el caso de los agentes policiales, el proceso de adaptación se da de manera constante, debido a los cambios de cargo o puestos que se da en el interior, el traslado o cambio de colocación a otras ciudades y de unidades policiales, que podrían afectar su adecuado desenvolvimiento, dando paso a situaciones o contextos estresantes. Esta situación para los efectivos policiales; se incrementa, además, por la crisis que afronta el sistema de salud, lo cual genera también estrés y pánico en la comunidad en general $\mathrm{y}$ otros profesionales debido al sentimiento de miedo al contagio y de fallecer a raíz del virus (Monterrosa-Castro et al., 2020). El estrés, es un conjunto de respuestas que tiene el ser humano desde la dimensión psicológica, física y conductual, las cuales se manifiestan frente a situaciones que son $y$ pueden ser interpretados como riesgo y/o amenaza (Lazarus \& Folkman, 1986).

En situaciones como en la que se está viviendo, Huang, Han, Luo, Ren y Zhou (2020) señalan que en promedio el 30\% de la población (230) que laboraba en el sector salud haciendo frente al COVID-19 presentan niveles altos de estrés. Hyunsunk, Hyeon, Yeong-Jun, Moran, JungAh, Juhee, Jeong-Ho (2016) también indicaron que los efectos que está dejando la presencia de COVID-19 en las personas, están ligados a la salud mental, como la depresión, ansiedad, estrés, entre otros.

\section{Método}

Diseño. El estudio ha sido desarrollado bajo el diseño descriptivo correlacional, siendo de corte transversal, presentando como objetivo establecer la relación existente entre la resiliencia y el estrés que presentan los efectivos policiales de la ciudad de Lima en tiempos de COVID19 y de forma complementaria se busca identificar como se encuentran los niveles en ambas variables, en hombres y mujeres.

Participantes. La muestra estuvo conformada por 408 policías que laboran en la ciudad de Lima, de los cuales 219 son varones, lo cual equivale a un 53.67\% y 189 mujeres que hacen un $46.32 \%$. Los participantes fueron agrupados, la edad promedio en los participantes es de 39 años y todos ellos laboran en la ciudad de Lima Metropolitana, con el servicio de patrullaje en vehículo motorizado mayor, menor y a píe. 
Materiales. En el presente estudio se han utilizado dos instrumentos de evaluación; el primero, fue la escala de resiliencia de 14 ítems (RS-14), el mismo que presenta un alfa de Cronbach de 0.79. Sánchez-Teruel y Robles-Bello (2015). Por otro lado, el instrumento de estrés, validado a través del criterio de tres jueces expertos, a su vez nos dio un alfa de Cronbach de .0912; los valores obtenidos nos revelan que ambos instrumentos presentan una adecuada consistencia interna.

Procedimiento. Debido a la situación que se vive en el país, los instrumentos fueron distribuidos de forma virtual; a través, de un enlace enviado a los teléfonos móviles de cada participante y siendo redistribuido con sus contactos inmediatos, provocando el muestreo bolo de nieve, que los llevaba a google formulario, para poder responder a los ítems planteados. Debemos señalar que, el instrumento solicitaba en un primer momento, el consentimiento a cerca de su participación y a su vez se le comunicaba en el mismo documento que este sería resuelto bajo el anonimato y se garantizaba el uso adecuado de la información. Posteriormente los datos recogidos, se extrajeron a un Excel para ser llevados al SPSS v25 y ser procesados acorde a los objetivos planteados.

\section{Resultados}

Los resultados de la investigación son presentados en las siguientes tablas a continuación:

Tabla 1

Relación de la actitud resiliente y el estrés en agentes policiales en Lima Metropolitana

\begin{tabular}{|c|c|c|c|c|c|c|}
\hline \multirow{2}{*}{\multicolumn{2}{|c|}{$\begin{array}{c}\mathrm{X}^{2}=31,359 ; \text { g.l. }= \\
4: p=0.002\end{array}$}} & & \multicolumn{2}{|c|}{ ESTRÉS } & \multirow[b]{2}{*}{ Alto } & \multirow{2}{*}{ Total } \\
\hline & & & Bajo & Medio & & \\
\hline \multirow{15}{*}{ 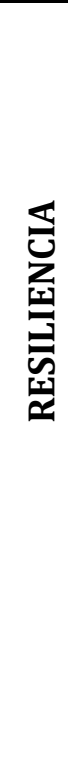 } & \multirow{4}{*}{ Bajo } & $\mathrm{f}$ & 1 & 2 & 98 & 101 \\
\hline & & $\%$ fila & $1 \%$ & $2 \%$ & $97 \%$ & $100 \%$ \\
\hline & & $\%$ columna & $1 \%$ & $2 \%$ & $40 \%$ & \\
\hline & & Res. Tip. & 0,0 & 0,1 & 2,2 & \\
\hline & \multirow{5}{*}{ Medio } & $\mathrm{f}$ & 4 & 49 & 111 & 164 \\
\hline & & $\%$ fila & $2 \%$ & $30 \%$ & $68 \%$ & $100 \%$ \\
\hline & & $\%$ columna & $7 \%$ & $50 \%$ & $45 \%$ & \\
\hline & & Res. Tip. & $-0,2$ & 1,2 & 1,8 & \\
\hline & & $\mathrm{f}$ & 56 & 48 & 39 & 143 \\
\hline & \multirow{4}{*}{ Alto } & $\%$ fila & $39 \%$ & $34 \%$ & $27 \%$ & $100 \%$ \\
\hline & & $\%$ columna & $92 \%$ & $48 \%$ & $16 \%$ & \\
\hline & & Res. Tip. & 1,8 & 1,1 & $-3,9$ & \\
\hline & & $\mathrm{f}$ & 61 & 99 & 248 & 408 \\
\hline & \multirow[t]{2}{*}{ Total } & $\%$ fila & $15 \%$ & $24 \%$ & $61 \%$ & $100 \%$ \\
\hline & & $\%$ columna & $100 \%$ & $100 \%$ & $100 \%$ & \\
\hline
\end{tabular}

Nota: Existe relación entre la actitud resiliente y el estrés, a mayor nivel de actitud resiliente, menor estrés. 


\section{Tabla 2}

Niveles de resiliencia en agentes policiales en Lima Metropolitana

\begin{tabular}{|c|c|c|c|}
\hline & & f & $\%$ \\
\hline \multirow{4}{*}{ 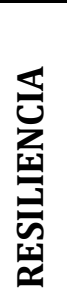 } & Bajo & 101 & $25 \%$ \\
\hline & Medio & 164 & $40 \%$ \\
\hline & Alto & 143 & $35 \%$ \\
\hline & Total & 408 & $100 \%$ \\
\hline
\end{tabular}

Nota. La actitud resiliente se muestra con mayor frecuencia en nivel medio y alto, en los agentes policiales

\section{Tabla 3}

Niveles de estrés en agentes policiales en Lima Metropolitana

\begin{tabular}{|c|c|c|c|}
\hline & & f & $\%$ \\
\hline \multirow{4}{*}{ 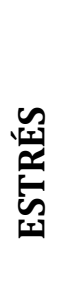 } & Bajo & 61 & $15 \%$ \\
\hline & Medio & 99 & $24 \%$ \\
\hline & Alto & 248 & $61 \%$ \\
\hline & Total & 408 & $100 \%$ \\
\hline
\end{tabular}

Nota. Predomina un nivel alto de estrés

\section{Tabla 4}

Niveles de estrés en agentes policiales hombres y mujeres de Lima Metropolitana

\begin{tabular}{cccccc}
\hline & \multicolumn{4}{c}{ SEXO } \\
\cline { 3 - 6 } & & \multicolumn{2}{c}{ Masculino } & \multicolumn{2}{c}{ Femenino } \\
\cline { 3 - 6 } & $\mathrm{f}$ & $\%$ & $\mathrm{~F}$ & $\%$ \\
\hline & Bajo & 50 & $23 \%$ & 11 & $6 \%$ \\
先 & Medio & 52 & $24 \%$ & 47 & $25 \%$ \\
& Alto & 117 & $53 \%$ & 131 & $69 \%$ \\
& Total & 219 & $100 \%$ & 189 & $100 \%$ \\
\hline
\end{tabular}

Nota. Existe mayor nivel de estrés en las mujeres 
Tabla 5

Niveles de resiliencia en agentes policiales hombres y mujeres de Lima Metropolitana

\begin{tabular}{|c|c|c|c|c|c|}
\hline & & \multicolumn{4}{|c|}{ SEXO } \\
\hline & & \multicolumn{2}{|c|}{ Masculino } & \multicolumn{2}{|c|}{ Femenino } \\
\hline & & $\mathrm{f}$ & $\%$ & $\mathrm{~F}$ & $\%$ \\
\hline \multirow{4}{*}{ 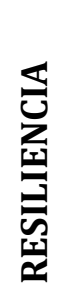 } & Bajo & 78 & $36 \%$ & 23 & $12 \%$ \\
\hline & Medio & 85 & $39 \%$ & 79 & $41 \%$ \\
\hline & Alto & 53 & $25 \%$ & 90 & $47 \%$ \\
\hline & Total & 219 & $100 \%$ & 189 & $100 \%$ \\
\hline
\end{tabular}

Nota. Existe mayor nivel de resiliencia en las mujeres

\section{Discusión}

Existen numerosos estudios sobre el estrés y la resiliencia en diversos grupos; sin embargo, no es lo mismo cuando se buscan estudios realizados en agentes policiales en la ciudad de Lima Metropolitana, tal como sucede en ciudades de otros países. El estudio nos demuestra que existe relación entre la resiliencia y el estrés, lo cual nos indica que a mayor resiliencia menor será la posibilidad de caer en niveles de estrés moderados o severos.

Los agentes policiales, desde su proceso de formación tienen conocimiento que una de sus labores más difíciles está destinada a luchar día a día contra la delincuencia, la violencia en todas sus manifestaciones, el de hacer respetar y que se cumplan las normas, entre ellas, el toque de queda, distanciamiento social y uso de mascarilla debido a la pandemia; además de su labor preventiva en el mantenimiento del orden público e interno; en donde su vida se encuentra muchas veces en riesgo, por lo que con razón, podríamos notar que el estrés negativo podría estar presente con facilidad en cada uno de sus miembros, siendo mermado por la capacidad resiliente que presentan.

Anderson, Libzenberger y Plecas (2002); McCraty y Atkinson (2012) señalan que, a nivel mundial, la labor policial es considera como una de las profesiones que genera más estrés, ocasionando en ellos síntomas a niveles físico, psicológico $\mathrm{y}$ conductual e incluso antes de participar en sus labores entendiéndose que un agente policial con estrés severo o crónico, podría ser candidato a cometer mayor número de errores en sus intervenciones policiales, los cuales son solo pueden perjudicarlo a él mismo, sino también a la ciudadanía.

Los factores que influyen en la determinación del estrés en los agentes policiales están mediados por los cambios de turno, condiciones laborales, clima laboral, respaldo institucional, entre otros. Gerber, Hartmann, Brand, HolsboerTrachsler \& Puhse (2010) ratifican que 
existe relación significativa entre los turnos laborales y los problemas sociales, generando descontento laboral y alteraciones de sueño.

Los resultados complementarios demuestran que las mujeres presentan altos niveles de estrés, mediado por niveles medios y altos de resiliencia, lo cual permite enfrentar las dificultades en la vida diaria y juega en papel muy importante en el agente policial y ello se relaciona con los estudios realizados sobre el estrés en mujeres, en su mayoría han indicado que son quienes presentan mayores índices de estrés moderado o severo a diferencia de los varones, García, Pérez y Luceño (2015) señalan a las mujeres como un grupo más vulnerable con altos niveles de estrés. Esto se relaciona con la carga familiar que también presentan.

Santos (2013) nos dice que la resiliencia "es la actitud vital positiva a pesar las circunstancias difíciles por las que atraviesa una persona, y representa el lado positivo de la salud mental" (p.12). Esto nos hace ver que la resiliencia se ha convertido en protector de los efectivos policiales, lo cual los ayuda a enfrentar las distintas situaciones adversas que se le presentan. Quizás sea un término para muchos agentes policiales o hasta para la misma institución nada o poco conocido, pero este estudio demuestra estar presente en ellos, contribuyendo en la búsqueda de su equilibrio y un buen desempeño.

De acuerdo a los resultados del presente estudio, la actitud resiliente de los agentes policiales de Lima Metropolitana en el contexto de la pandemia, ha presentado un nivel medio y alto, representada por un $40 \%$ y $35 \%$ respectivamente. Indicativo que muestra que dicho personal a pesar de la situación de miedo y estrés que origina esta enfermedad, se sobreponen a ella. Tirino-Quijije \& Giniebra-Urra (2020), señalaron que la resiliencia constituye un factor protector en situaciones de estrés como los originados por el COVID-19, coincidiendo con Averill, Averill, Kelmendi Albdallah y Southwick (2018) indican que la resiliencia es un regulador eficaz de la respuesta al estrés que presenta una persona. Asimismo, Moreno, Cárdenas, Cárdenas, Nieto \& Lopera (2021) en su estudio manifiestan que mejorar la capacidad resiliente mejora el manejo del estrés.

La pandemia ha traído consigo un conjunto de factores psicológicos como el miedo a contraer el virus, el medio a fallecer, la ansiedad y el estrés. Estos factores han sido el común denominador que ha presentado personal del sector público así como otros factores de índole social (estrés laboral) como producto del COVID-19 . Sin embargo, frente a estas situaciones ambientales los agentes policiales de Lima Metropolitana han tenido una respuesta positiva (resiliente), conforme lo demuestran los resultados del presente estudio.

\section{Conclusiones}

La situación actual, que vive el mundo entero por la presencia del COVID-19 afecta a muchos de los seres humanos, en especial a aquellos que se encuentran en primera línea para frenar la ola de contagios. Como ya lo habíamos mencionado, la institución 
policial del Perú a comparación de otras instituciones públicas, sigue siendo una de las más golpeadas por la pandemia. Los agentes policiales, no solo se enfrentan ante la inseguridad ciudadana que se vive; ahora se suma el control de la ciudadanía, para evitar los contagios, según las ordenes que brinda el gobierno, lo cual ha generado variaciones del servicio policial, afectando la inestabilidad emocional, dando paso a un nivel alto de estrés, que tiene que ser moderado por la capacidad resiliente, la constante en este ejercicio, podría desgastar al personal policial, por lo cual, se considera oportuno que la institución empoderé a su personal, con la finalidad de potenciar esas características resilientes, que le permitan enfrentar de manera

\section{Referencias}

Águila, A., Calcines, M. Monteagudo de la Guardia, R. \& Nieves, A. (2015). Estrés académico. Revista Edumecentro, 7(2), 163-178 http://scielo.sld.cu/scielo.php?scri pt $=$ sci arttext\&pid $=$ S207728742015000200013

Anderson, G., Libzenberger, R. \& Plecas, P. (2002). Physical evidence of police oficcer stress. Policing: An International Journal of Police Strategies \& Management 25(2), 399-420.

https://www.ojp.gov/library/abstr acts/physical-evidence-policeofficer-stress

Averill, L., Averill, C., Kelmendi, B., Albdallah, Ch. \& Southwick, S. (2018). Stress response modulation underlying the psychobiology of resilience. Current Psychiatry exitosa cada situación laboral que se les pueda presentar.

No obstante, a pesar del nivel alto de estrés presentado por los agentes policiales de Lima Metropolitana, se ha mostrado un nivel medio y alto en la actitud resiliente de dicho personal frente a la pandemia. Lo que evidencia determinadas capacidades en parte de esta población para disminuir los riesgos de vulnerabilidad, adaptándose a los cambios de la coyuntura existente y superar las situaciones desfavorables o adversidades que trae consigo el COVID-19.

Reports, 20(4), 27. https://doi.org/10.1007/s11920$\underline{\text { 018-0887-x }}$

Banco Mundial (2020). Resumen anual 2020: el impacto de la COVID-19. https://blogs.worldbank.org/es/vo ices/resumen-anual-2020-elimpacto-de-la-covid-19coronavirus-en-12-graficos

Becoña, E. (2006). Resiliencia: Definición, características y utilidad del concepto. Revista de psicopatología y psicología clínica, 11(3), 125-146. https://doi.org/10.5944/rppc.vol.1 1.num.3.2006.4024

Constitución. (1993). Constitución Política del Perú (Editora Perú (ed.)). Editora Perú.

El Peruano. (2020). Decreto Supremo No 044-2020-PCM. Diario Oficial El 
Peruano,

$10-12$ www.gob.pe/mindef

El Peruano (2020). Decreto supremo $N^{\circ} 083$ 2020-PCM, que prorroga el Estado de Emergencia Nacional por las graves circunstancias que afectan la vida de la Nación a consecuencia del COVID-19 y estable otras disposiciones.

https://busquedas.elperuano.pe/n ormaslegales/decreto-supremoque-prorroga-el-estado-deemergencia-naciona-decretosupremo-n-083-2020-pcm1866214-1/

García, Pérez y Luceño (2015). Turnos y estrés psicosocial en los policías locales de Madrid. Estrés y ansiedad. 21(1), 57-70. https://dialnet.unirioja.es/servlet/ articulo?codigo $=6850075$

Gerber, M., Hartmann, T., Brand, S., Holsboer-Trachsler, E., \& Pühse, U. (2010). The relationship between shift work, perceived stress, sleep and health in Swiss police officers. Journal of Criminal Justice, 38(6), 1167-1175.

https://doi.org/10.1016/j.jcrimjus. $\underline{2010.09 .005}$

Huang J, Han M, Luo T, Ren A \& Zhou X. (2020). Mental health survey of 230 medical staff in a tertiary infectious disease hospital for COVID-19. Revista China de Salud Laboral y Enfermedades Profesionales, $38(3)$. https://dx.doi.org/10.3760/cma.j.c $\underline{\text { n121094-20200219-00063 }}$

Hyunsunk, J., Hyeon, Y., Yeong-Jun, S., Moran, K., Jung-Ah, M., Juhee, Ch. y Jeong-Ho, Ch. (2016). Mental health status of people isolated due to Middle East Respiratory Syndome.
Epidemiol Health, 8.https://doi.org/10.4178/epih.e2 016048

IDL. (2020). Frente a la pandemia. https://www.ilo.org/wcmsp5/gro ups/public/---ed_protect/--protrav/--safework/documents/publication/ wcms_742732.pdf

Marquina-Luján, R. (2017). Estilos educativos parentales y resiliencia en adolescentes de la Institución Educativa Pública "Colegio Mayor Secundario Presidente del Perú". Revista ConCiencia EPG, 2(2), 20-27.

https://doi.org/10.32654/CONCIE NCIAEPG.2-2.1

McCraty, R. Atkinson, M. (2012). Resilence training program reduces physiological and psychological stress in police officers. Global Advances in Health and Medicine, 1(5),

44-66.

https://doi.org/10.7453/gahmj.20 $\underline{12.1 .5 .013}$

Monterrosa-Castro, A., Dávila-Ruiz, R., Mejía-Mantilla, A., ContrerasSaldarriaga, J., Mercado-Lara, M., \& Florez-Monterrosa, C. (2020). Estrés laboral, ansiedad y miedo al COVID-19 en médicos generales colombianos. MedUNAB, 23(2), 195-213.

https://doi.org/10.29375/012370 $\underline{47.3890}$

Moreno, J., Cárdenas, C., Cárdenas, J., Nieto J. \& Lopera J. (2021). Impacto del entrenamiento en resiliencia $\mathrm{y}$ manejo del estrés: estudio de caso en la Policía Nacional de Colombia. Revista Logos Ciencia \& Tecnología, 13(1),

96-115. 
https://doi.org/10.22335/rlct.v13i 1.1323

Organización Mundial de la Salud. (2021). Preguntas y respuestas sobre la enfermedad por coronavirus (COVID-19). Organización Mundial de La Salud. https://www.who.int/es/emergen cies/diseases/novel-coronavirus2019/advice-for-public/q-acoronaviruses?gclid=Cj0KCQiAgom BBhDXARIsAFNyUqOHBNRBe4Z9125mViA2heBSvSpvzOuM5Ixk QsFQY04u9GMmd9fjCUaAtFMEAL $\underline{\mathrm{w} w C B}$

Policía Nacional del Perú (08 de febrero del 2021). Boletín Diario: Operaciones. Cuadros de acciones y atenciones diarias del personal ETRSP PNP COVID-19. Equipo de trabajo de la red de seguridad del personal PNP. ETRSP PNP COVID-19. Ministerio del interior

Rutter, M. (2007). Implications of resilience concepts for scientific understanding. Annals of the New York Academy of Sciences, 1094(1), 1-12. https://doi.

org/10.1196/annals.1376.002

Sánchez-Teruel, D, \& Robles-Bello, M. (2015). Escala de Resiliencia 14 ítems (RS-14): Propiedades psicométricas de la versión en español. Revista Iberoamericana de Diagnóstico y Evaluación - e Avaliação Psicológica, 2(40),103113.

https://www.redalyc.org/articulo. oa?id=4596/459645432011

Santos, R. (2013). Levantarse y luchar: Cómo superar la adversidad con la resiliencia. Conecta Edición.

Tirino-Quijije, D. A., \& Giniebra-Urra, R. (2020). Resiliencia Y Ansiedad En Personas Con Covid-19 En Manta, Manabí, Ecuador. Revista Científica Multidisciplinaria Arbitrada "Yachasun," 4(7), 323-343. https://doi.org/10.46296/yc.v4i7. $\underline{0052}$ 